PROCEEDINGS OF THE

AMERICAN MATHEMATICAL SOCIETY

Volume 138, Number 3, March 2010, Pages 1101-1111

S 0002-9939(09)09844-X

Article electronically published on October 23, 2009

\title{
REVERSIBILITY OF A SIMPLE RANDOM WALK ON PERIODIC TREES
}

\author{
SERDAR ALTOK \\ (Communicated by Richard C. Bradley)
}

\begin{abstract}
We show that if $\mu$ is a reversible measure for simple random walk on rooted trees whose branches are covers of finite connected directed graphs, then $\mu$ is supported on rooted covers of finite connected undirected graphs. For a given finite connected directed graph $G$ and a cover $T$ of $G$, we give an algorithm to determine whether there exists a finite connected undirected graph whose cover has a branch isomorphic to $T$.
\end{abstract}

\section{INTRODUCTION}

The speed of a random walk $\left\langle X_{n}\right\rangle$ on a tree is defined to be $\lim _{n \rightarrow \infty} \frac{\left|X_{n}\right|}{n}$ whenever this limit exists, where $\left|X_{n}\right|$ is the graph distance of the location of the random walker after $n$ moves to the initial location of the random walker.

To study the speed, it is useful to introduce a random walk on (isomorphism classes of) rooted trees. The root will typically represent the location of the random walker in which we are interested. Let $(T, o)$ denote the tree $T$ rooted at the vertex $o$ and $[T, o]$ denote the rooted isomorphism class of $(T, o)$. If the walker is at $[T, o]$, then the next state is $\left[T, o^{\prime}\right]$ with positive probability for each neighbor $o^{\prime}$ of $o$.

The speed of simple random walk on Galton-Watson trees was studied by Lyons, Permantle, Peres (1995a) and Takacs (1997), where the latter deals with periodic trees which are deterministic multi-type Galton-Watson trees. (In the rest of this paper, we shall write LPP for short for Lyons, Pemantle, Peres.)

If $T$ has the distribution of a Galton-Watson tree with offspring distribution $\left\{p_{k}\right\}_{k \in \mathbb{N}}$, then $T$ is infinite with positive probability when $\sum k p_{k}>1$, and in this case simple random walk on $T$ is transient almost surely given that $T$ is infinite (see Collevecchio (2006) for a short proof of this fact). In LPP (1995a), it was shown that the speed of simple random walk on $T$ is a constant number almost surely, and an explicit formula was given for the speed. This was done by finding a reversible stationary measure on rooted trees, an augmented Galton-Watson measure, which is concentrated on rooted trees with the following property: if a uniformly chosen edge incident to the root is deleted, then the component of the root has the same distribution as $T$. With a little work, stationarity of an augmented Galton-Watson measure gives the speed on a tree that has the distribution of an augmented GaltonWatson tree, and the property mentioned above implies that this speed coincides with the speed on $T$.

Received by the editors October 3, 2008, and, in revised form, November 26, 2008.

2000 Mathematics Subject Classification. Primary 60J10; Secondary 60G50.

(C)2009 American Mathematical Society Reverts to public domain 28 years from publication 
We use the word graph to mean a finite connected graph that could possibly be directed or undirected. The word tree can mean both a finite or an infinite tree.

A formula for the speed of biased random walk, in particular simple random walk, on periodic trees (covers of strongly connected directed graphs, hence deterministic multi-type Galton-Watson trees) was first given by Takacs (1997). If $G$ is a strongly connected directed graph, Takacs found a stationary measure $\mu$ for a biased random walk on rooted trees with the property that there is an edge incident to the root such that when that edge is deleted, the component of the root is a cover of $G$. This property implies that the speed on any cover of $G$ is the same as the speed on a tree $T$ with distribution $\mu$, which Takacs calculated by using the stationarity of $\mu$.

The existence of a reversible measure for simple random walk is useful in two ways: It is easier to determine a reversible stationary measure than a stationary measure, and it implies that there exists a unimodular measure with the same support, which implies many other results through the mass transport principle (Aldous, Lyons (2007)).

The stationary measure of Takacs is not necessarily reversible. Examples of reversible measures for simple random walk on rooted trees, where the deletion of an edge (in fact any edge) incident to the root leaves the root in a cover of a directed graph, can be obtained in the following way: The uniform measure on the vertex set of an undirected graph $H$ lifts to a measure on rooted covers of $H$, and biasing this measure by the degree of the root gives a reversible measure for simple random walk (LPP $(1995 \mathrm{~b}))$. Thus if $T$ is a cover of a directed graph, $T^{\prime}$ is the cover of an undirected graph and there is an edge $e$ of $T^{\prime}$ such that when $e$ is deleted, one of the two remaining components is isomorphic to $T$, then the calculation of the speed of simple random walk on $T$, which is equal to the speed on $T^{\prime}$, is straightforward.

If $\mu$ is a reversible measure for simple random walk on a directed graph $G$, then $G$ necessarily comes from an undirected graph; that is, there is an undirected graph $H$ such that if each edge of $H$ is replaced by two edges oriented in opposite directions, then simple random walk on the resulting graph has the same state space and transition probabilities as the walk on $G$. A similar statement holds for simple random walk on trees with branches that are covers of directed graphs: Our main theorem (Theorem 3.7) says that if $\mu$ is a reversible measure for simple random walk on rooted trees with the property that there is an edge incident to the root whose deletion leaves the root in a cover of a directed graph, then $\mu$-a.s. $(T, o)$ is a rooted cover of an undirected graph.

The relationship described above between reversible measures for simple random walk on rooted covers of undirected graphs and the measures obtained by biasing them by the degree of the root is a special case of the relationship between a reversible measure for simple random walk and the corresponding unimodular measure. A measure $\mu$ on rooted trees is reversible for simple random walk if and only if $\mu$, biased by the reciprocal of the degree of the root, is unimodular (Aldous, Lyons (2007)). In this context our result says that the only unimodular rooted trees that have periodic branches are the ones mentioned above.

There are several related open problems. One is finding unimodular random networks with subtrees that have the distribution of a non-deterministic multi-type Galton-Watson tree. Other open problems about simple and biased random walks on Galton-Watson trees can be found in LPP (1995b). 


\section{THE BASICS}

Definitions and notation. Let $[n]:=\{1, \ldots, n\}$. The word graph always refers to a finite connected graph that can be directed or undirected. Trees can be both finite or infinite.

For a graph $G$, let $V(G)$ and $E(G)$ denote the set of vertices of $G$ and the set of edges of $G$ respectively. For $x, y \in V(G)$, we write $x \sim y$ if $x$ and $y$ are connected by an edge. If $G$ is a directed graph and $x$ and $y$ are connected by a directed edge that is oriented from $x$ to $y$, let $e_{x y}$ denote that edge. If $G$ is an undirected graph and $x \sim y$, then $e_{x y}=e_{y x}$ is the edge connecting $x$ and $y$. We shall occasionally write $e_{x, y}$ instead of $e_{x y}$. For any graph $G$, if $e \in E(G)$, we write $e \sim x$ if $x$ is an endpoint of $e$.

For a directed graph $G$ and $x, y \in V(G)$, a path from $x$ to $y$ is a sequence of edges $e_{1}, \ldots, e_{n-1} \in E(G)$ such that there exist $v_{1}=x, v_{2}, \ldots, v_{n}=y \in V(G)$ such that for all $i \in[n-1]$, we have $e_{i}=e_{v_{i}, v_{i+1}}$. We say that a directed graph $G$ is connected if for each pair of vertices $x$ and $y$ there is a directed path from $x$ to $y$ or from $y$ to $x$. A directed graph $G$ is strongly connected if for each pair of vertices $x$ and $y$ there are directed paths from $x$ to $y$ and from $y$ to $x$. Thus every strongly connected graph is connected.

For an undirected graph $G$ and $x, y \in V(G)$, a path between $x$ and $y$ is a sequence of edges $e_{1}, \ldots, e_{n-1} \in E(G)$ such that there exist $v_{1}=x, v_{2}, \ldots, v_{n}=$ $y \in V(G)$ such that for all $i \in[n-1], e_{i}=e_{v_{i}, v_{i+1}}$. A path is called simple if each vertex on the path is visited exactly once. Let $\operatorname{deg}_{G} x$ denote the degree of $x$, i.e. the number of edges coming out of $x$. We shall omit the subscript $G$ in $\operatorname{deg}_{G} x$ whenever it is clear what $G$ is.

A tree is a connected graph that does not contain any cycles. Hence, for a tree $T$ and $x, y \in V(T)$, there is a unique simple path between $x$ and $y$. Let $e_{x}(y)$ denote the first edge on the simple path from $x$ to $y$. For $f \in E(T)$, let $e_{x}(f)$ be the first edge of the shortest path that starts from $x$ and contains $f$. For all $x \in V(T)$ and all $e \in E(T)$ such that $e \sim x$, let $C_{x, e}$ denote the component of $x$ when $e$ is deleted. We shall call $\left(C_{x, e}, x\right)$ a branch of $T$. Thus when an edge of a tree is deleted, we get two branches.

Let $G, G^{\prime}$ be two graphs that are both directed or undirected. We say $G$ is isomorphic to $G^{\prime}$ if there are bijections $\phi_{V}: V(G) \rightarrow V\left(G^{\prime}\right)$ and $\phi_{E}: E(G) \rightarrow$ $E\left(G^{\prime}\right)$ such that for all $x \sim y \in V(G)$, we have $\phi_{E}\left(e_{x y}\right)=e_{\phi_{V}(x), \phi_{V}(y)}$. In this case we write $G \cong G^{\prime}$. For $o \in V(G)$ and $o^{\prime} \in V\left(G^{\prime}\right),(G, o)$ is rooted isomorphic to $\left(G^{\prime}, o^{\prime}\right)$ if $G \cong G^{\prime}$ with a bijection satisfying $\phi_{V}(o)=o^{\prime}$. In this case we write $(G, o) \cong\left(G^{\prime}, o^{\prime}\right)$. We denote the rooted isomorphism class of $(G, o)$ by $[G, o]$. We define the degree of the root of $[G, o]$ to be the degree of the root of any representative of $[G, o]$.

For a vertex $x$ of a directed graph $G$, let $T_{x}$ be the (unrooted) tree whose vertices are finite paths (on $G$ ) starting from $x$, where two vertices are connected by an undirected edge if one is the extension of the other by one edge in $G$. For all $x, y \in V(G)$, we have that $(G, x) \cong(G, y)$ if and only if $\left(T_{x}, x\right) \cong\left(T_{y}, y\right)$. Any tree isomorphic to $T_{x}$ for some $x \in G$ is called a cover of $G$.

For an undirected graph $H$, we define covers of $H$ in the following way: Fix a vertex $x$ of $H$ and consider the unrooted tree $T_{x}$ whose vertices are finite paths on $H$ that start from $x$ and do not backtrack, where two vertices are connected by an edge if one is the extension of the other by one edge. It is easy to see that for all 

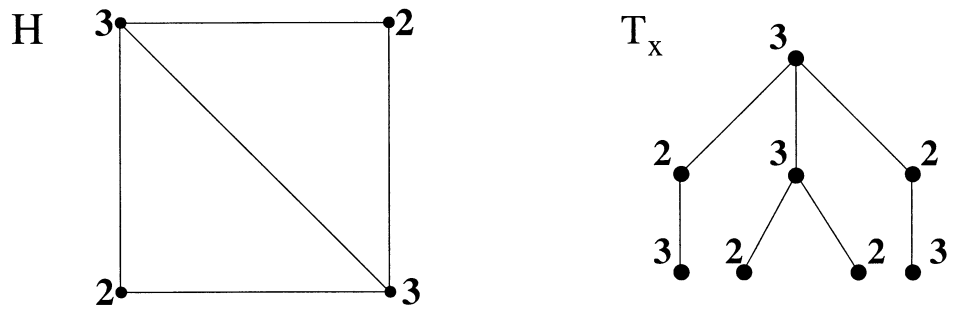

Figure 1. $H$ and its cover $T_{x}$, where $x$ is either of the two vertices labeled 3 .

$x, y \in V(H)$, we have $T_{x}$ is isomorphic to $T_{y}$. Any tree that is isomorphic to $T_{x}$ for some $x \in V(H)$ is called a cover of $H$.

In Figure 1 we give an example of a finite undirected graph $H$ and part of its cover $T_{x}$, where $x$ is either of the two vertices of $H$ that are labeled 3 . In $T_{x}$ the very top vertex corresponds to the path that consists of $x$, the vertices at level one correspond to the paths that start at $x$ and that have length 1 , and so on.

A rooted cover, $(T, o)$, of a strongly connected directed graph is called a periodic tree. A tree $(T, o)$ is periodic if and only if there is an $N \in \mathbb{N}$ such that for each $x \in V(T)$ there exists a vertex $y$ whose graph distance to $o$ is less than $N$ and for $e=e_{x}(o)$ and $f=e_{y}(o)$, we have that $\left(C_{x, e}, x\right)$ is rooted isomorphic to $\left(C_{y, f}, y\right)$. All periodic trees are deterministic multi-type Galton-Watson trees with finitely many types; that is, there is a labeling of the vertices by elements of a finite set $L$ such that for every $y, z \in V(T)$ that have the same label, we have that for all $m \in L$, $y$ and $z$ have an equal number of children whose label is $m$. (See Athreva. Nerv (2004) for a definition of multi-type Galton Watson trees in general.)

Reversible measures on covers of undirected graphs. In this subsection we construct some reversible measures for simple random walk on rooted covers of any undirected graph $H$ by studying a corresponding random walk on $\{[H, x]: x \in$ $V(H)\}$ and show that the branches of covers $H$ are periodic trees.

For all $x \in V(H)$ and $o \in V\left(T_{x}\right)$, define $h(o)$ to be the last vertex of the path on $H$ that represents $o$. If $y \in V(H)$ and $o^{\prime} \in V\left(T_{y}\right)$, we have that $\left(T_{x}, o\right) \cong\left(T_{y}, o^{\prime}\right)$ if and only if $(H, h(o)) \cong\left(H, h\left(o^{\prime}\right)\right)$. Thus each $\left[T_{x}, o\right]$ is identified with some $[H, y]$, and via this identification a random walk on isomorphism classes of rooted covers of $H$ gives a random walk on $\{[H, x]: x \in V(H)\}$, and vice versa. Another consequence is that each cover of $H$ is quasi-transitive; that is, $\left\{\left[T_{x}, o\right]: o \in V\left(T_{x}\right)\right\}$ is finite.

We consider simple random walk on isomorphism classes of rooted covers of $H$; that is, for $x \in V(H)$ and $o, o^{\prime} \in V\left(T_{x}\right)$ if the walker is at $\left[T_{x}, o\right]$, in the next step of the random walk the walker moves to $\left[T_{x}, o^{\prime}\right]$ with probability $k / \operatorname{deg}_{T_{x}} o$, where $k$ is the cardinality of the set $\left\{o^{\prime \prime} \in V\left(T_{x}\right): o^{\prime \prime} \sim o,\left(T_{x}, o^{\prime \prime}\right) \cong\left(T_{x}, o^{\prime}\right)\right\}$.

Let $\nu$ be the uniform measure on $V(H)$. Define the measure $\mu$ by $\mu([H, x]):=$ $\sum \nu(y)$, where the sum runs over all $y \in V(H)$ such that $(H, y) \cong(H, x)$. This induces a measure on isomorphism classes of rooted covers of $H$, which we also denote by $\mu$ : We define $\mu\left(\left[T_{x}, o\right]\right)$ to be $\mu([H, h(o)])$. Biasing each of the measures $\mu$ by the degree of the respective root within the underlying graph or tree (i.e. the degree of the root of any representative from the isomorphism class), we get two 


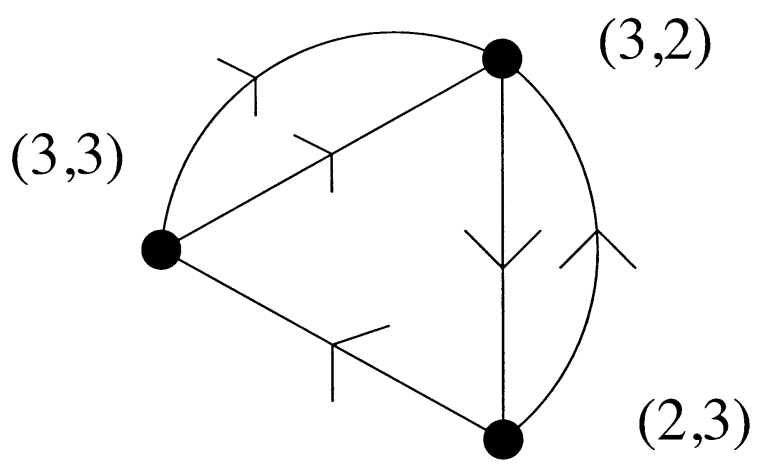

Figure 2. $H^{\prime}$ for the graph $H$ in Figure 1 .

new measures that are reversible for simple random walk on the spaces on which they are defined.

As we mentioned before, the branches of covers of undirected graphs are periodic trees. To see this, let $H$ be an undirected graph. For all $x \in V(H)$, a directed graph $H^{\prime}$ whose rooted covers are isomorphic to $\left\{\left(C_{o, e}, o\right): o \in V\left(T_{x}\right), o \sim e \in E\left(T_{x}\right)\right\}$ can be constructed as follows:

Label the vertices of $H$ by elements of a finite set $L$ such that for all $x, y \in V(H)$ we have that $(H, x) \cong(H, y)$ if and only if $x$ and $y$ have the same label. For all $x \in V(H)$, let $l(x)$ denote the label of $x$. Let $V\left(H^{\prime}\right):=\{(l(x), l(y)): x, y \in$ $V(H), x \sim y\}$ and connect every $\left(l_{1}, l_{2}\right),\left(l_{2}, l_{3}\right) \in V\left(H^{\prime}\right)$ by $m-\delta_{l_{1}, l_{3}}$ edges oriented from $\left(l_{1}, l_{2}\right)$ to $\left(l_{2}, l_{3}\right)$, where $m$ is the number of edges between any two vertices of $H$ labeled $l_{2}$ and $l_{3}$, and $\delta_{l_{1}, l_{3}}=1$ if $l_{1}=l_{3}$ and $\delta_{l_{1}, l_{3}}=0$ otherwise.

If $H$ is as in Figure 1, then $L=\{2,3\}$ and $V\left(H^{\prime}\right)=\{(2.3),(3,2),(3,3)\}$. There will be exactly one edge directed from $(3,2)$ to $(2,3)$ in the corresponding $H^{\prime}$, since the only way to extend a path on $H$ whose last two vertices have labels 3 and 2 in that order is by adding an edge whose other endpoint has label 3. The complete graph $H^{\prime}$ can be seen in Figure 2 .

Thus, as we mentioned in the introduction, the stationary measures described above enable us to calculate the speed of simple random walk on any cover of a directed graph that is a branch of a cover of some undirected graph. Figure 2 shows $H^{\prime}$ for the undirected graph $H$ illustrated in Figure 1 .

\section{THE MAIN THEOREM}

In the previous section we showed that if $H$ is an undirected graph, then there is a reversible measure for simple random walk on rooted covers of $H$ and the branches of rooted covers of $H$ are deterministic multi-type Galton-Watson trees. Now our aim is to show that those are the only reversible measures on trees with the following property: For each vertex $x$ of the tree there is an edge $e \sim x$ such that when $e$ is deleted the branch that $x$ is in is a cover of a directed graph.

Let $R$ be a collection of rooted covers of directed graphs.

Let $A:=\{[T, o]:(T, o) \in R\}$.

Let $D:=\{[T, o]: o \in V(T),[T, x] \in A$ for some $x \in V(T)\}$.

Let $\mathbf{E}:=\left\{[T, o]: o \in V(T), \forall x \in V(T) \exists e \in E(T)\right.$ such that $\left.e \sim x,\left[C_{x, e}, x\right] \in A\right\}$.

Let $\Pi:=D \cup \mathbf{E}$. 
We show that if $\mu$ is a reversible measure for simple random walk on $\Pi$, then $\mu$-a.s. $(T, o)$ is the cover of an undirected graph.

Note that $R$ is at most countable, and even when $R$ is finite, the sets $D, \mathbf{E}$ and consequently $\boldsymbol{\Pi}$ are not necessarily finite.

Remark 3.1. For any $(T, o) \in R$ if $(T, o)$ is a cover of $G^{\prime}$, then for any $y \in V(T)$ we have that $\left(C_{y^{\prime}, e_{y^{\prime}}(o)}, y^{\prime}\right)$ is a cover of $G^{\prime}$. In fact there is a directed graph $G$ such that $(T, o)$ is a cover of $G$, and if $\left(T^{\prime}, o^{\prime}\right)$ is any cover of $G$, then there exists $y^{\prime} \in V(T)$ such that $\left(T^{\prime}, o^{\prime}\right)$ is isomorphic to $\left(C_{y^{\prime}, e_{y^{\prime}}(o)}, y^{\prime}\right)$.

Let

$$
A^{\prime}:=\left\{\left[C_{x, e}, x\right]: x \in V(T) \text { for some }(T, o) \in R, e=e_{x}(o)\right\} .
$$

Since the trees in $R$ are covers of directed graphs, $A^{\prime}$ consists of isomorphism classes of all covers of the directed graphs chosen as in Remark 3.1. When $R$ is finite, since the directed graphs that the trees in $R$ cover have finitely many types, $A^{\prime}$ is finite. When $R$ is countable, $A^{\prime}$ is also countable.

Remark 3.2. By definition of $A^{\prime}$ if $\left[C_{x, e}, x\right] \in A^{\prime}$, then for all $y \in V\left(C_{x, e}, x\right)$ we have $\left[C_{y, e_{y}(x)}, y\right] \in A^{\prime}$ as well.

Remark 3.3. If $[T, o] \in \mathbf{\Pi}-\mathbf{E}$, then $\left(T, o^{\prime}\right) \in R$ for some $o^{\prime} \in V(T)$. Consequently $\left(T, o^{\prime}\right)$ is the cover of a directed graph. This implies that for any $x \in V(T)$ such that $o^{\prime} \neq x$, we have $\left[C_{x, e_{x}\left(o^{\prime}\right)}, x\right] \in A^{\prime}$.

For $[T, o] \in \Pi$, let

$$
E_{T}:=\left\{e \in E(T): e=e_{x y}, x \sim y,\left[C_{x, e}, x\right] \in A^{\prime},\left[C_{y, e}, y\right] \in A^{\prime}\right\} .
$$

We shall also use $E_{T}$ to denote the graph whose edge set is $E_{T}$ and whose vertex set is $\left\{x \in V(T): \exists e \in E_{T}, x \sim e\right\}$.

Lemma 3.4. For all $[T, o] \in \Pi, E_{T}$ is connected.

Proof. Let $e=e_{x y}, f=f_{z t} \in E_{T}$ with $x \sim y, z \sim t$ being distinct vertices of $T$. Without loss of generality assume $f \in C_{y, e}$ and $e \in C_{z, f}$. Since $e \in E_{T}$, $\left[C_{y, e}, y\right] \in A^{\prime}$. Let $p \sim r$ be two vertices on the simple path between $e$ and $f$, with $p$ being closer to $e$ than to $f$. Let $d=e_{p r}$. Since $\left[C_{y, e}, y\right] \in A^{\prime}$, by Remark 3.2 we have $\left[C_{r, d}, r\right] \in A^{\prime}$. By the same reasoning (using $f$ instead of $e$ ), we get $\left[C_{p, d}, p\right] \in A^{\prime}$. These two together give that $d \in E_{T}$. Since $d$ is an arbitrary edge on the simple path between $e$ and $f$, all the edges on the simple path between $e$ and $f$ are in $E_{T}$, which implies that $E_{T}$ is connected.

Lemma 3.5. Assume $R$ is finite. Then, if $[T, o] \in \Pi$ satisfies $E_{T}=E(T), T$ is a cover of an undirected graph $H$.

Proof. Assume that $E_{T}=E(T)$. For each vertex $x \in T,\left\{\left[C_{y, e_{x, y}}, y\right]: y \sim x\right\}$ has at most $\operatorname{deg} x$ elements, which are all isomorphism classes of covers of a directed graph $G$. Since $R$ is finite, we have that $\operatorname{deg} x$ is bounded and there is a finite number of such graphs $G$, where each of them has a finite number of covers up to isomorphism. Consequently, $\{[T, o]: o \in T\}$ is finite, and therefore $T$ is quasitransitive. Let $H$ be the undirected graph whose vertex set is $\{[T, o]: o \in T\}$, where two vertices $[T, o]$ and $\left[T, o^{\prime}\right]$ are connected if there exists $o^{\prime \prime} \in V(T)$ such that $o^{\prime \prime} \sim o$ and $\left(T, o^{\prime \prime}\right) \cong\left(T, o^{\prime}\right)$. Since $T$ is quasi-transitive and connected, $H$ is finite and connected. It is easy to see that $T$ is a cover of $H$. 
Lemma 3.6. Assume $R$ is finite. Let $\left[T, o^{\prime \prime}\right] \in \Pi$ satisfy $\emptyset \neq E_{T} \neq E(T)$. Then if $\mu$ is a reversible measure for simple random walk on $\boldsymbol{\Pi}$ such that $\mu([T, x]: x \in$ $V(T))=1, T$ is a cover of an undirected graph.

Proof. Note that in the lemma $T$ is fixed, but we get different rooted trees by allowing the root to be any vertex of $T$. Assume that $\mu$ is as in the statement of the lemma.

Now $E_{T}$ is connected by Lemma 3.4. For each $e \in E(T)$ that is not in $E_{T}$, if the deletion of $e$ gives two infinite components, then orient $e$ towards $E_{T}$. Otherwise there is no orientation on $e$. Since $\mu$ is reversible for simple random walk, the measure $\mu^{\prime}$ obtained by biasing $\mu$ by the reciprocal of the degree of the root is unimodular; i.e. the mass transport principle holds for $\mu^{\prime}$ (Aldous, Lyons (2007)). Therefore we can apply the mass transport principle in the following way: Send mass one through every oriented edge. We have

$$
\sum_{x, o \in V(T)} f(T, o, x) \mu^{\prime}([T, o])=\sum_{x, o \in V(T)} f(T, x, o) \mu^{\prime}([T, o]),
$$

where $f(T, x, y)$ is the mass sent from $x$ to $y$.

If the mass is sent from $o$ (along $e_{o}$ ), then since $C_{o, e_{o}}$ is infinite, $o$ receives mass from some vertex $y$ through the edge $e_{y}$. By the same reasoning we can conclude that the root receives mass if it sends mass. Thus for every $[T, o]$, the mass received by $o$ is greater than or equal to the mass sent by $o$. Hence the mass the root sends is equal to the mass the root receives $\mu^{\prime}$-almost surely. Let

$$
C_{0}:=\left\{[T, o]: e^{\prime} \sim o \sim e_{o x} \text { for some } e_{o x} \notin E_{T}, e^{\prime} \in E_{T}, f(T, x, o)=1\right\} .
$$

Thus for each $[T, o] \in C_{0}$, o has at least one neighbor $x$ such that when $e_{o x} \notin E_{T}$ is deleted, we get two infinite branches. If $[T, o] \in C_{0}$, then $f(T, o, x)=0$ for all $x \in V(T)$ since $E_{T}$ is connected. Consequently we have that for each $[T, o] \in C_{0}$

$$
\sum_{x \in T} f(T, o, x)=0<1 \leq \sum_{x \in T} f(T, x, o) .
$$

Since the expected mass the root sends is equal to the expected mass the root receives, this implies $\mu^{\prime}\left(C_{0}\right)=0$, which is equivalent to $\mu\left(C_{0}\right)=0$.

For $x, y \in V(T)$, let $d(x, y)$ be the number of edges on the shortest path between $x$ and $y$. For all $n \geq 1$, let

$$
C_{n}:=\left\{[T, o]: o, o^{\prime} \in V(T), d\left(o, o^{\prime}\right)=n,\left[T, o^{\prime}\right] \in C_{0}\right\} .
$$

Since $\mu$ is stationary and $\mu\left(C_{0}\right)=0$, we have $\mu\left(C_{n}\right)=0$ for all $n \in \mathbb{N}$. Thus $0=\mu\left(\bigcup_{n=0}^{\infty} C_{n}\right)=\mu([T, x]: x \in V(T))=1$, which is a contradiction. Therefore when $E_{T}$ is deleted, the remaining components are finite.

We claim that $\left\{\left[C_{x, e}, x\right]: x \in V(T), x \sim e \in E(T)\right\}$ is finite. The set $\left\{\left[C_{x, e}, x\right]:\right.$ $\left.x \in V(T), x \sim e \in E_{T}\right\}$ is finite since it is a subset of $A^{\prime}$. When $f \notin E_{T}$ is deleted, we get two branches: one finite branch and one infinite branch whose edge set contains $E_{T}$. When an edge in $E_{T}$ is deleted, by definition we get two branches in $A^{\prime}$, one of which contains $f$. Therefore by Remark 3.2 the finite branches are all in $A^{\prime}$. In particular there is a finite number of finite branches up to isomorphism. Next we show that there is a finite number of infinite branches up to isomorphism obtained by deleting an edge $f \notin E_{T}$ as well, and this will prove our claim.

Let

$$
E_{0}:=\left\{e_{x y}: x \sim y \in V(T) \text { such that } e_{x y} \notin E_{T} \text { and } \exists f \in E_{T}, f \sim x\right\} .
$$


So, $E_{0}$ is the set of edges $e=e_{x y}$ that are not in $E_{T}$ but incident to an edge $f \in E_{T}$ where $f \sim x \sim e$ and $y$ is not the endpoint of any edge in $E_{T}$.

For $e_{x y} \in E_{0}$, if $e=e_{x z} \sim x$, then either $e \in E_{T}$ or $\left(C_{z, e}, z\right)$ is finite. In either case $\left[C_{z, e}, z\right] \in A^{\prime}$. Since $A^{\prime}$ is finite and $\operatorname{deg}_{T} x$ is bounded (since $R$ is finite), we have that $\left\{[T, x]: \exists y \in V(T), e_{x y} \in E_{0}\right\}$ is finite. This implies that $\left\{\left[C_{x, e_{x y}}, x\right]: e_{x y} \in E_{0}\right\}$ is finite.

Now if $f=e_{z t}$ is any edge not in $E_{T} \cup E_{0}$ and $\left[C_{z, f}, z\right]$ is the infinite branch when $f$ is deleted, then let $e=e_{x y}$ be the unique element of $E_{0} \cap E\left(C_{z, f}\right)$ that is of minimal distance to $f$. Now let $\left(T_{f, z}, z\right)$ be the finite tree that is obtained from $\left(C_{z, f}, z\right)$ by deleting all the edges that are also in $E\left(C_{x, e}\right)$. Thus if we identify $x \in V\left(T_{f, z}\right)$ with $x \in V\left(C_{x, e}\right)$, we get $\left(C_{z, f}, z\right)$.

Since there is a finite number of finite branches up to isomorphism, the set $\left\{\left[T_{f, z}, z\right]: f=e_{z t} \in E(T)-\left(E_{T} \cup E_{0}\right)\right\}$ is finite. We have already shown that $\left\{\left[C_{x, e_{x y}}, x\right]: e_{x y} \in E_{0}\right\}$ is finite. These two together imply that $\left\{\left[C_{z, f}, z\right] ; f=e_{z t} \in\right.$ $E(T)-\left(E_{T} \cup E_{0}\right), V\left(C_{z, f}\right)$ is not finite $\}$ is finite.

This proves our claim that $\left\{\left[C_{x, e}, x\right]: x \in V(T), x \sim e \in E(T)\right\}$ is finite. As in the proof of Lemma 3.5. since $\operatorname{deg}_{T} x$ is bounded, this implies that $T$ is the cover of an undirected graph.

Theorem 3.7. Let $\mu$ be a measure on $\Pi$. If $\mu$ is reversible for simple random walk, then $\mu$-a.s. $(T, o)$ is a cover of an undirected graph $H$.

Proof. To show that the conclusion holds, it is enough to prove it for extremal unimodular measures, since any unimodular measure is a mixture of extremal unimodular measures.

So assume $\mu$ is extremal. By Theorem 4.7 of Aldous. Lyons (2007), the sets invariant under unrooted isomorphisms have $\mu$-measure 1 or 0 .

Let

$$
B:=\left\{[T, o] \in \Pi: o \in V(T), E_{T} \neq \emptyset\right\} .
$$

Since $R$ is countable, $B$ is countable. By Theorem 4.7 of Aldous, Lyons (2007), $B$ has measure 1 or 0 . If $\mu(B)=1$, then since $B$ is countable, we have $\mu(\{[T, o]: o \in$ $V(T)\})=1$ for some labeled tree $T$. Now let $e=e_{x y} \in E_{T}$ so that $\left(C_{x, e}, x\right),\left(C_{y, e}, y\right)$ are covers of some directed graphs $G_{1}$ and $G_{2}$. Then every $z \in V(T)$ has the property that there is an $f \in E(T)$ incident to $z$ (namely $e_{z}(x)$ or $e_{z}(y)$ ) such that $\left(C_{z, f}, z\right)$ is a cover of $G_{1}$ or $G_{2}$. Thus we can apply Lemma 3.5 and Lemma 3.6 with $R$ being the set of all covers of $G_{1}$ and $G_{2}$ to conclude that $T$ is a cover of an undirected graph.

We now show that $\mu\left((\boldsymbol{\Pi}-\mathbf{E}) \cap B^{c}\right)=\mu\left((D-\mathbf{E}) \cap B^{c}\right)=0$. Theorem 4.7 of Aldous, Lyons (2007) implies that $\mu(D-\mathbf{E})=0$ or $\mu(D-\mathbf{E})=1$. If the latter were true, then since $D$ is countable, we would have $\mu([T, o]: o \in V(T))=1$ for some $[T, o] \in D-\mathbf{E}$. Then by Remark 3.3 we would have $\left[T, o^{\prime}\right] \in A$ for some $o^{\prime} \in V(T)$. If there are two such vertices, then the last statement of Remark 3.3 implies that $E_{T} \neq \emptyset$ and consequently $[T, o] \in B$. Hence if $[T, o] \in(D-\mathbf{E}) \cap B^{c}$ there is a unique vertex $x_{T}$ such that $\left[T, x_{T}\right] \in A$, and stationarity of $\mu$ implies $\mu\left((D-\mathbf{E}) \cap B^{c}\right)=0$.

Let $B_{1}:=B^{c} \cap \mathbf{E}$. To complete the proof we need to show that if $\mu(B)=0$, then $\mu$-a.e. $[T, o] \in B_{1}$ is a rooted cover of an undirected graph.

Now assume $\mu(B)=0$. Let $[T, o] \in B_{1}$. We first show that there is a bijection between the vertices and edges of $T$ to obtain an orientation of the edges. In this case, for every $x \in T$, there is exactly one edge incident to $x$ such that when 
that edge is deleted, the component of $x$ rooted at $x$ is isomorphic to a tree in $R$, since if there were two such edges $e=e_{x y} \neq f$, we would have $\left[C_{x, e}, x\right] \in A$ and $\left[C_{x, f}, x\right] \in A$; then $e=e_{y x} \in\left[C_{x, f}, x\right]$ and consequently $\left[C_{y, e}, y\right] \in A^{\prime}$. (See Remark [3.2.) Combining this with $\left[C_{x, e}, x\right] \in A \subset A^{\prime}$, we would have $e \in E_{T}$, which is a contradiction.

For all $x \in V(T)$, let $e_{x}$ be the edge that satisfies $\left[C_{x, e_{x}}, x\right] \in A$. This map is one-to-one since if $e_{x}=e_{y}$, then we necessarily have $x \sim y$ and $e_{x}=e_{y}=e_{x y} \in E_{T}$, which is a contradiction. It is onto since if $e=e_{x y}$ for some $x \sim y$ and $e_{x} \neq e \neq$ $e_{y}$, then since $[T, o] \in \mathbf{E}, \exists f \sim x, f \neq e$ such that $\left[C_{x, f}, x\right] \in A$, which implies $\left[C_{y, e}, y\right] \in A^{\prime}$. Using the same argument but reversing the positions of $x$ and $y$, we get $\left[C_{x, e}, x\right] \in A^{\prime}$. This together with $\left[C_{y, e}, y\right] \in A^{\prime}$ gives that $e \in E_{T}$, contradicting that $E_{T}=\emptyset$. This proves our claim.

We are ready to apply the mass transport principle. As in the proof of Lemma 3.6. $\mu^{\prime}$ is unimodular. For all $[T, o] \in B_{1}$ and all $x \in V(T)$, orient $e_{x}$ away from $x$. Start with a unit mass at each vertex and transport it along the oriented edges whose deletion leaves two infinite components. If mass is sent from $x$ (along $e_{x}$ ), then since $C_{x, e_{x}}$ is infinite, $x$ receives mass from some vertex $y$ through the edge $e_{y}$. By the same reasoning we can conclude that each vertex receives mass if and only if it sends mass. For any tree $T \in B_{1}$, let $S_{T}$ be the set of vertices of $T$ through which mass is transported. The mass transport principle implies that $\mu^{\prime}$-almost surely (hence $\mu$ ) if the root is in $S_{T}$; then the root has exactly two neighbors that are in $S_{T}$. Stationarity of $\mu$ implies that any vertex in $S_{T}$ has exactly two neighbors that are in $S_{T}$.

Let $S_{T}^{\prime}$ be the graph whose vertex set is $S_{T}$ and $E\left(S_{T}^{\prime}\right):=\left\{e_{x}: x \in S_{T}\right\}$. Then $S_{T}^{\prime}$ is connected: For if $S_{T}^{\prime}$ had two disconnected components, they would necessarily be two copies of $\mathbb{Z}$. Then the deletion of any edge of $T$ that is on the simple path between the two components would give two infinite components. This would imply that all the vertices on that path are in $S_{T}$. Therefore the vertices where the path joins either component of $S_{T}^{\prime}$ would have three neighbors that are in $S_{T}$, contradicting the fact that any vertex in $S_{T}$ has exactly two neighbors that are in $S_{T}$.

Thus $\mu^{\prime}$-a.s. or equivalently $\mu$-a.s. $(T, o)$ has exactly one copy of $\mathbb{Z}$, namely $S_{T}^{\prime}$; has finite branches that are attached to $S_{T}^{\prime}$; and with respect to the orientation of the edges described above, every edge of $S_{T}^{\prime}$ is oriented in the same direction and the edges that are not in $S_{T}^{\prime}$ are oriented towards $S_{T}^{\prime}$. Let $B_{2}$ be the set of isomorphism classes of such rooted trees.

For any $(T, o) \in R$ define

$$
C_{T, o}:=\left\{\left[T^{\prime}, o^{\prime}\right] \in B_{2}: \exists x \in V(T)\left(C_{x, e_{x}}, x\right) \cong(T, o)\right\}
$$

Since $R$ is countable there are countably many sets of the form $C_{T, o}$, and by Theorem 4.7 of Aldous, Lyons (2007) each of them is $\mu$-trivial. For any $(T, o),\left(T^{\prime}, o^{\prime}\right) \in$ $R$, write $\left(T^{\prime}, o^{\prime}\right) \subset(T, o)$ if there exists $x \in V(T)$ such that $\left(C_{x, e_{x}(o)}, x\right) \cong\left(T^{\prime}, o^{\prime}\right)$. We have $C_{T, o} \subset C_{T^{\prime}, o^{\prime}}$ if $\left(T^{\prime}, o^{\prime}\right) \subset(T, o)$ with equality when $(T, o) \subset\left(T^{\prime}, o^{\prime}\right)$ as well. Also $C_{T, o} \cap C_{T^{\prime}, o^{\prime}}=\emptyset$ if and only if neither $\left(T^{\prime}, o^{\prime}\right) \subset(T, o)$ nor $(T, o) \subset\left(T^{\prime}, o^{\prime}\right)$ holds. Thus $\mu\left(C_{T, o}\right)=\mu\left(C_{T^{\prime}, o^{\prime}}\right)=1$ holds if and only if we have $C_{T, o} \subset C_{T^{\prime}, o^{\prime}}$ or $C_{T^{\prime}, o^{\prime}} \subset C_{T, o}$. This gives a total ordering on the set $K:=\left\{C_{T, o}: \mu\left(C_{T, o}\right)=1\right\}$. Then $\mu\left(\bigcap_{C_{T, o} \in K} C_{T, o}\right)=1$. 
Let $[T, o] \in \bigcap_{C_{T^{\prime}, o^{\prime}} \in K} C_{T^{\prime}, o^{\prime}}$. Fix $y \in V(T)$. We consider two cases: If there exists $C_{T^{\prime}, o^{\prime}} \in K$ such that there are infinitely many $x \in V(T)-V\left(C_{y, e_{y}}\right)$ such that $\left(C_{x, e_{x}}, x\right) \cong\left(T^{\prime}, o^{\prime}\right)$, then $T$ is a cover of an undirected graph. To see this, fix two such vertices $x_{1}, x_{2}$. Without loss of generality assume $\left(C_{x_{2}, e_{x_{2}}}, x_{2}\right) \subset\left(C_{x_{1}, e_{x_{1}}}, x_{1}\right)$. Now we delete the edges of $\left(C_{x_{1}, e_{x_{1}}}, x_{1}\right)$ that are also in $\left(C_{x_{2}, e_{x_{2}}}, x_{2}\right)$ to get a graph where the degree of $x_{2}$ is 1 . Identify $x_{1}$ with $x_{2}$ to get the undirected graph $H$. It is easy to see that $T$ is a cover of $H$.

Otherwise for each $\left(T^{\prime}, o^{\prime}\right) \in R$, there are finitely many $x \in V(T)-V\left(C_{y, e_{y}}\right)$ such that $\left(T^{\prime}, o^{\prime}\right) \cong\left(C_{x, e_{x}}, x\right)$. We claim that there could be only one such $T$ up to isomorphism: Let $\left[T_{1}, o_{1}\right] \in \bigcap_{C_{T, o} \in K} C_{T, o}$ have the property that there exists (equivalently for all) $y_{1} \in V\left(T_{1}\right)$ such that for each $\left(T^{\prime}, o^{\prime}\right) \in R$, there are finitely many $x \in V\left(T_{1}\right)-V\left(C_{y_{1}, e_{y_{1}}}\right)$ with $\left(T^{\prime}, o^{\prime}\right) \cong\left(C_{x, e_{x}}, x\right)$. We define an isomorphism $h: T \rightarrow T_{1}$ by determining $h(x)$ for each $x \in V(T)$. Fix $x \in V(T)$ and let $x^{\prime} \in V(T)$ be such that $\left(C_{x, e_{x}}, x\right) \cong\left(C_{x^{\prime}, e_{x^{\prime}}}, x^{\prime}\right)$ and there exists no $x^{\prime \prime} \in V(T)-V\left(C_{x^{\prime}, e_{x^{\prime}}}\right)$ such that $\left(C_{x^{\prime}, e_{x^{\prime}}}, x^{\prime}\right) \cong\left(C_{x^{\prime \prime}, e_{x^{\prime \prime}}}, x^{\prime \prime}\right)$. Since $\left[T_{1}, o_{1}\right] \in \bigcap_{C_{T^{\prime}, o^{\prime}} \in K} C_{T^{\prime}, o^{\prime}}$ there exists $y^{\prime} \in V\left(T_{1}\right)$ such that $\left(C_{y^{\prime}, e_{y^{\prime}}}, y^{\prime}\right) \cong\left(C_{x^{\prime}, e_{x^{\prime}}}, x^{\prime}\right)$ and there exists no $y^{\prime \prime} \in V\left(T_{1}\right)-$ $V\left(C_{y^{\prime}, e_{y^{\prime}}}\right)$ such that $\left(C_{y^{\prime}, e_{y^{\prime}}}, y^{\prime}\right) \cong\left(C_{y^{\prime \prime}, e_{y^{\prime \prime}}}, y^{\prime \prime}\right)$. By using the isomorphism between $\left(C_{x^{\prime}, e_{x^{\prime}}}, x^{\prime}\right)$ and $\left(C_{y^{\prime}, e_{y^{\prime}}}, y^{\prime}\right)$ we determine the image of $x$. It is easy to see that $h$ is well-defined and is an isomorphism. To see that $\mu[T, o]=0$ for all $o \in V(T)$, fix $\left(T^{\prime}, o^{\prime}\right) \in R$ such that there exists $x \in V(T)$ with $\left(T^{\prime}, o^{\prime}\right) \cong\left(C_{x, e_{x}}, x\right)$. As discussed before there is a unique vertex $x^{\prime} \in V(T)-V\left(C_{x, e_{x}}\right)$ such that $\left(C_{x^{\prime}, e_{x^{\prime}}}, x^{\prime}\right) \cong\left(T^{\prime}, o^{\prime}\right)$ and there exists no vertex $x^{\prime \prime} \in V(T)-V\left(C_{x^{\prime}, e_{x^{\prime}}}\right)$ with $\left(T^{\prime}, o^{\prime}\right) \cong\left(C_{x^{\prime \prime}, e_{x^{\prime \prime}}}, x^{\prime \prime}\right)$. By stationarity of $\mu$, we have that $\mu[T, o]=0$ for all $o \in V(T)$. Therefore the first case holds $\mu$-a.s., and in that case we have shown that $T$ is a cover of an undirected graph.

Finally, we describe a method to determine whether for a given tree $(T, o)$ that is a rooted cover of a directed graph, there is an undirected graph whose cover has a branch (or equivalently infinitely many branches) isomorphic to $(T, o)$.

Fix $(T, o)$ as above and let $R=\{(T, o)\}$. Now if $H$ is a finite undirected graph such that for some fixed cover $T_{H}$ of $H$ and for some $e=e_{x y} \in E\left(T_{H}\right)$ we have $(T, o)$ is isomorphic to $\left(C_{x, e}, x\right)$, then $A^{\prime}=F_{H}$, where

$$
F_{H}:=\left\{\left[C_{x, e}, x\right]: x \in V\left(T_{H}\right), e \in E\left(T_{H}\right), e \sim x\right\} .
$$

Hence we try to find an undirected graph $H$ for which $A^{\prime}=F_{H}$.

Given two disjoint rooted trees $\left(T_{1}, o_{1}\right),\left(T_{2}, o_{2}\right)$, define $\left[\left(T_{1}, o_{1}\right) \bullet\left(T_{2}, o_{2}\right)\right]$ to be the tree that is formed by joining $o_{1}$ and $o_{2}$ by an edge and that is rooted at $o_{1}$.

Let $S$ be the set of rooted trees $\left(T^{\prime}, o^{\prime}\right)$ such that if $\left(T^{\prime}, o^{\prime}\right)=\left[\left(T_{1}, o_{1}\right) \bullet\left(T_{2}, o_{2}\right)\right]$, then $\left[T_{1}, o_{1}\right]$ and $\left[T_{2}, o_{2}\right]$ are both in $A^{\prime}$. If $S$ is non-empty, then let $H^{\prime}$ be the graph whose vertices are the elements of $S$, where $\left(T^{\prime}, o^{\prime}\right),\left(T^{\prime \prime}, o^{\prime \prime}\right) \in V\left(H^{\prime}\right)$ are connected by an edge if there exists $x \in V\left(T^{\prime}\right)$ such that $x \sim o^{\prime}$ and $\left(T^{\prime}, x\right) \cong\left(T^{\prime \prime}, o^{\prime \prime}\right)$. Since $(T, o)$ is the cover of a finite directed graph, the sets $A^{\prime}, S$ and $H^{\prime}$ are all finite. If $H^{\prime}$ has a connected component $H$ with the following properties:

i) $\forall\left(T^{\prime}, o^{\prime}\right) \in V(H), \operatorname{deg}_{H^{\prime}}\left(T^{\prime}, o^{\prime}\right)=\operatorname{deg}_{T^{\prime}} o^{\prime}$,

ii) $A^{\prime}=\left\{\left[T_{1}, o_{1}\right]: \exists\left(T_{2}, o_{2}\right)\right.$ such that $\left.\left[\left(T_{1}, o_{1}\right) \bullet\left(T_{2}, o_{2}\right)\right] \in V(H)\right\}$, then we have $F_{H}=A^{\prime}$.

Conversely, if there is an undirected graph $K$ such that $[T, o]=\left[C_{x, e}, x\right]$ for some $x \in V\left(T_{K}\right)$ and $e \in E\left(T_{K}\right)$, then $H^{\prime}$ has a connected component $H$ that satisfies 
conditions $i$ ) and $i i$ ). Hence it is enough to check whether $H^{\prime}$ has a connected component $H$ that satisfies conditions $i$ ) and $i i$ ).

\section{ACKNOWLEDGEMENT}

The author is grateful to Russ Lyons for his suggestions, discussions and continuous support throughout the preparation of this paper.

\section{REFERENCES}

Aldous, D., Lyons, R. (2007). Processes on Unimodular Random Networks. Electron. J. Probab. 12, Paper 54 (2007), 1454-1508. MR2354165 (2008m:60012)

Athreya, K. B., Ney, P. (2004). Branching Processes. Dover, Mineola, NY. MR2047480

Collevecchio, A. (2006). On the Transience of Processes Defined on Galton-Watson Trees. Ann. Probab. 34, No. 3, 870-878. MR2243872 (2007k:60264)

Lyons, R., Pemantle, R., Peres, Y. (1995a). Ergodic Theory on Galton-Watson Trees: Speed of Random Walk and Dimension of Harmonic Measure. Ergodic Theory Dynam. Systems 15, 593-619. MR.1336708 (96e:60125)

Lyons, R., Pemantle, R., Peres, Y. (1995b). Unsolved Problems Concerning Random Walks on Trees. Classical and Modern Branching Processes, 223-238, Krisna B. Athreya and Peter Jagers, eds., Springer, New York, 1997. MR1601753 (98j:60098)

Takacs, C. (1997). Random Walk on Periodic Trees. Electron. J. Probab. 2, 1-16. MR:1436761 (97m:60101)

Department of Mathematics, Indiana University, Bloomington, Indiana 47405

E-mail address: saltok@umail.iu.edu 